\title{
Struttura-azione di poesia e narratività nella scrittura di Vincenzo Consolo
}

\author{
Maria Attanasio
}

Scrittrice

\section{Abstract}

Alla ricerca di un'espressività nuova rispetto all'omologazione linguistica della contemporaneità, la scrittura di Vincenzo Consolo assume le modalità ritmiche e la densità metaforica della poesia: una vera e propria struttura-azione testuale che fa interferire la lingua della memoria e la memoria della lingua, la sequenzialità del tempo narrativo e la verticalità di quello della poesia.

Parole chiave: viaggio, storia, parola, coro, metafora, poesia.

\section{Abstract}

In search of a new means of expression with respect to linguistic approval of contemporaneity, the writing of Vincenzo Consolo takes on the rhythmic formal procedures and metaphorical density of poetry: a true and actual textual structuration that makes language get in the way of memory and the memory of language, the sequential nature of narrative time and the verticality of poetic time.

Key words: journey, history, word, heart, metaphor, poetry.

Benchè tutti i suoi libri siano traboccanti di citazioni, epigrafi e continui riferimenti a poeti d'ogni luogo e tempo - da Omero a Teocrito, ad Ariosto, a Iacopo da Lentini, a Shakespeare, a Leopardi, a D'Annunzio, a Dante, e a tanti altri - Vicenzo Consolo non ha mai pubblicato un libro di poesie, a differenza degli altri scrittori contemporanei siciliani - Sciascia, Addamo, Bufalino, Bonaviri, D’Arrigo, ad esempio - la cui narrativa è stata spesso affiancata o preceduta da un'autonoma produzione poetica.

Eppure è poeta, il più poeta tra i narratori siciliani; non si tratta di una generica liricità che crocianamente trasborda in ogni genere, ma di una testualità che, dentro le sequenze del tempo narrativo dei romanzi, e in quelle argomentative della saggistica, fonde libertà espressiva e referenzialità compositiva, ragioni etiche e motivazioni estetiche, ideologia e parola: immaginifica interazione tra la lingua della memoria, che restituisce il passato come metafora 
del presente, e la memoria della lingua, che, immergendosi nella lievitante stratificazione culturale e mitica delle parole, restituisce significatività al linguaggio; esemplare è in questo senso un racconto appartenente a Le pietre di Pantalica, I linguaggi del bosco, in cui lo scrittore racconta l'esperienza di un fanciullo che, durante un soggiorno in campagna, apprende dalla selvatica amica Amalia ad ascoltare il bosco - il suono del vento tra gli alberi, il gorgoglio dell'acqua, l'oscurata parola delle bestie - e a rinominare con una lingua argotica ogni cosa

Fu lei a rivelarmi il bosco, il bosco più intricato e segreto. Mi rivelava i nomi di ogni cosa, alberi, arbusti, erbe, fiori, quadrupedi, rettili, uccelli, insetti... E appena li nominava, sembrava che in quel momento esistessero. Nominava in una lingua di sua invenzione, una lingua unica e personale, che ora a poco a poco insegnava a me e con la quale per la prima volta comunicava. ${ }^{1}$

metafora della necessità di una espressività nuova in una contemporaneità che, in nome del mercato e del profitto, opera, anche a livello linguistico, una marginalizzazione dei valori di un mondo a carattere antropocentrico. E in questi ultimi anni più che mai: la definizione di "guerra umanitaria» ad esempio, o l'umanizzazione di borse e mercati che «tremano, soffrono, si esaltano, si deprimono, sono euforiche», ecc., mentre, ridotti a puri beni strumentali dell'economia, gli uomini sono diventati semplici «risorse», talvolta da «rottamare».

Vincenzo Consolo ha sempre respinto la piatta orizzontalità della lingua — quella da lui definita "tecnologica-aziendale» che, diffusa dai mezzi di informazione, rende afasica la realtà — spingendo invece la sua prosa a contaminarsi con altri generi, soprattutto con la verticalità immaginativa e demercificata del linguaggio poetico. La poesia è infatti oggi l'unica tra le arti che non diventa merce, perché il suo linguaggio, traboccando sempre dalla pura formulazione linguistica, non può mai totalmente identificarsi con quello della comunicazione: "coscienza anticipante», rispetto ai valori del proprio tempo, ma anche coscienza critica nei confronti del linguaggio del proprio tempo. La sua ricerca espressiva si muove perciò verso una scrittura che sia, insieme, esperienza di verità - non di semplice realtà - e testimonianza di libertà.

Verità della storia — nella storia — e libertà della parola — nella parola - costituiscono infatti la struttura ideologicamente portante della sua narratività, che nella metafora del viaggio — presente e centrale in tutti i suoi libri — si congiungono, corrispondendo spesso — il viaggio e la scrittura — all'inizio e alla fine dello sviluppo narrativo; tappe necessarie di un processo conoscitivo che, coniugando storia ed esistenza, diventa presa di coscienza, parola, il cui nucleo — insieme motivazione e finalità — è sempre l'uomo, "Prima viene la vita, — scrive in Retablo - quella umana, sacra, inoffendibile, e quindi ogni altro: filosofia, scienza, arte, poesia, bellezza...». ${ }^{2}$ 
Tra il primo romanzo, La ferita dell'aprile (Mondadori, 1963) e l'ultimo, Lo spasimo di Palermo (Mondadori, 1999), il rapporto tra storia e parola - e quindi il senso della metafora del viaggio — si modifica però profondamente. Se fino a Retablo il viaggio è avventura conoscitiva e utopia, che dilatano il tempo, ingannano la morte - la causa vera del viaggio, per Fabrizio Clerici, il protagonista di questo libro, «è lo scontento del tempo che viviamo, della nostra vita, di noi, e il bisogno di staccarsene, morirne, e vivere nel sogno dell'ere passate, antiche, che nella lontananza ci figuriamo d'oro, poetiche, come sempre è nell'irrealtà dei sogni, sogni intendo come sostanza de' nostri desideri ${ }^{3}$, nella produzione successiva perde ogni connotazione conoscitiva, diventa nostos, amara verifica in un "paese piombato nella notte», «nell'Europa deserta di ragione»; Chino Martinez, il protagonista de Lo Spasimo di Paler$m o$, ha infatti la consapevolezza che ogni viaggio è «tempesta, tremito, perdita, dolore, incontro e oblio, degrado, colpa sepolta rimorso, assillo senza posa». ${ }^{4}$

La modificazione del senso della storia modifica anche quello della parola, con un'accentuazione sempre più espressiva ed espressionista del suo linguaggio, e con un utilizzo in funzione narrativamente destrutturante, rispetto a un tradizionale concetto di romanzo; un utilizzo, in questo senso, presente fin dalle prime opere.

In un saggio del 1997, Il sorriso dell'ignoto marinaio vent'anni dopo, che conclude la raccolta di saggi $A l$ di qua dal faro, scrive:

Il suo linguaggio e la sua struttura volevano indicare il superamento in senso etico, estetico, attraverso mimesi, parodie, fratture, spezzature, oltranze immaginative, dei romanzi d'intreccio dispiegati e dominati dall'autore, di tutti i linguaggi logici, illuministci, che nella loro limpida, serena geometrizzazione escludevano le voci dei margini. ${ }^{5}$

Una vera e propria «struttura-azione» di poesia potentemente interviene a costituire il corpo stesso della narratività di Vincenzo Consolo, restringendo gli spazi di comunicazione, dissolvendo ogni ordinata sequenzialità di tempi e di sintassi, travalicando ogni rigida separazione tra i generi; ed emergendo in punte espressive - disancorate dalla narrazione - con due difformi e spesso simultanei riporti: tragico nei confronti della storia, lirico nei confronti della natura; una dimensione, quest'ultima, vissuta quasi con un senso di imbarazzo dalla coscienza etica e ideologica dell'autore, che ne teme la smemorante e avvolgente bellezza fuori dalla storia.

Lo scrittore sente però fortemente il malioso richiamo che da essa proviene: la staniante fascinazione di forme, suoni, segni, paesaggi che, intrecciandosi o alternandosi agli echi drammatici e stridenti della storia, come una sorta di respiro profondo attraversa tutta la sua scrittura; non si tratta di una schi-

3. Ibid., p. 177.

4. Op. cit., p. 98.

5. Vincenzo Consolo, Di qua dal faro, Milano: Mondadori, 2001, p. 282. 
zofrenia espressiva, ma di una strutturale complementarietà, come complementari - all'interno di una complessiva visione del mondo - sono in Dante e Leopardi, filosofia e sentimento, ideologia e poesia. E non è un caso che entrambi, insieme a Omero, a Eliot e a Lucio Piccolo, siano i poeti di riferimento più presenti nella sua scrittura, che assume talvolta il carattere di una visionaria riscrittura, pulsante di echi, parole, reperti linguistici, risalenti dal fondo del già scritto.

In una sorta di rarefatto silenzio leopardiano, ne $\mathrm{Il}$ sorriso dell'ignoto marinaio - mentre il bastimento con il Mandralisca e tutto il suo carico di storia ed esistenze, approda davanti alla Rocca di Tindari — risuona, al di sopra dei tempi e degli spazi della narrazione, la voce visionaria dello scrittore a richiamare dal buio dei millenni Adelasia, la solitaria badessa centenaria, che torna ad aggirarsi tra le celle ormai disabitate, a interrogare invano l'immemore fluire

Quindi Adelasia, regina d'alabastro, ferme le trine sullo sbuffo, impassibile attese che il convento si sfacesse. Chi è, in nome di Dio? - di solitaria badessa centenaria in clausura domanda che si perde per le celle, i vani enormi, gli anditi vacanti. - Vi manda l'arcivescovo? -

E fuori era il vuoto. Vorticare di giorni e soli e acque, venti a raffiche, a spirali, muro d'arenaria che si sfalda, duna che si spiana, collina, scivolio di pietra, consumo. Il cardo emerge, si torce, offre all'estremo il fiore tremulo, diafano per l'occhio cavo dell'asino bianco. Luce che brucia, morde, divora lati spigoli contorni, stempera toni macchie, scolora. Impasta cespi, sbianca le ramaglie, oltre la piana mobile di scaglie orizzonti vanifica, rimescola le masse. ${ }^{6}$

Lo stesso cosmico smarrimento dei sensi e dell'intelletto lo restituisce attraverso il protagonista di Retablo, che, sul colle di Segesta, davanti alla grandiosità dell'arte e della natura, vede spalancarsi le inaudite voragini del tempo:

Sedetti su lo stilòbate, fra le colonne, sotto l'architrave da cui pendeva e oscillava al vento il cappero, il rovo, l'euforbia, a contemplare il deserto spazio, ascoltare il silenzio spesso su codesto luogo. Un silenzio ancora più smarrente per lo strider delle gazze, dei corvi, che neri sopra il cielo del tempio e sopra il vuoto della gran voragine grevi volteggiano, per frinire lungo di cicale e il gorgogliare dell'acque del Crinisio o Scamandro che dall'abisso, eco sopra eco, sonora si levava.

finendo con la letterale citazione di un verso de L'Infinito leopardiano. «E sedendo e mirando...».

C’è una circolarità geografica nella scrittura consoliana: gli stessi luoghi - esemplari di una perduta bellezza, come i resti della grecità, o di una aggrovigliata contemporaneità, come la Palermo barocca, o la tennologica

6. Vincenzo Consolo, Il sorriso dell'ignoto marinaio, Torino: Einaudi, 1976, p. 8.

7. Op. cit., p. 79. 
Milano - ritornano ciclicamente nelle sue opere; Segesta tornerà infatti a essere rappresentata ne L'olivo e l'olivastro:

E là, nel centro, nel recinto aperto in ogni lato, verso ogni punto, esposto alla notte dell'estate che porta sulle brezze odori arsicci di fieni, nepitelle, porta le scansioni del silenzio, del buio, strida, pigolii, sprazzi verdastri, gialli, luccichii, aperto in alto all'infinito spazio, mi pongo arreso, supino, e vado, $\mathrm{mi}$ perdo nella lettura stupefatta del libro immenso, in incessante mutamento, nella scritura abbagliante delle stelle, dei soli remoti, dei chiodi tremendi del mistero.....Rimango immobile e contemplo, sprofondo estatico nei palpiti, nei fuochi, nei bagliori, nei frammenti incandescenti che si staccano, precipitano filando, si spengono, finiscono nel più profondo nero. ${ }^{8}$

Un testo di una straordinaria intensità poetica; l'immagine di quei «chiodi tremendi del mistero" resta, ad esempio, ostinata a perciare la mente: l'enigma dell'improvviso ritrovarsi esistenti nel tempo.

Interminabili potrebbero essere le citazioni, tratte da tutti i suoi libri, a esemplificazione della variegata e lirica restituzione della natura; del paesaggio soprattutto, colto in tutte le ore e le stagioni: dalla campagna alla città al mare, la cui plurale valenza simbolica nella sua scrittura meriterebbe una trattazione a parte; nella lunga e illuminante intervista, pubblicata ne La fuga dall'Etna (Donzelli, 1993), lo scrittore definisce infatti il mare, come un luogo di invasione e possessione della natura, dell'esistenza, dei miti e dei simboli: simboli ambigui, spesso, insieme, di valori opposti, stridenti come la vita e la morte.

Ma la percezione del luogo nella sua scrittura non è mai orizzontale e univoca: nella sua spazialità affiorano memorie, miti, scritture, esistenze, visioni; e non solo nei luoghi dell'arte o in quelli della storia, ma anche in quelli abituali e scontati del quotidiano, come, ne Lo spasimo di Palermo, la stanza di un albergo:

Scricchiolii, la guida scivolosa nella curva e il fiato di sempre venefico e pungente, mai tarme blatte, mai topi in quest'ammasso d'alberi e fasciami disarmati, forse madrepore, alghe secolari, fermenti sigillati di mari tropicali, lo sciabordio è nelle tubature verniciate, nel rigagnolo sotto il marciapiede. L'angustia è forzata dagli specchi, uguale gentilezza è impressa su carta tende copriletto, lo stridore è nel giallo degli eterni girasoli. ${ }^{9}$

A volte è invece il sentimento a far scattare la tensione lirica, privilegiatamente l'amore nelle sue diverse espressioni: come dolcissima e lacerante coniugalità ne Lo Spasimo di Palermo, come sconvolgente passionalità in Retablo, il meno storico dei suoi romanzi, benchè ci sia anche in questo, come in tutta la sua produzione, una precisa lettura etica e di classe; nucleo motivante è, in 
questo libro, l'esistenza, nel suo rapporto, a volte conflittuale, di ragione e sentimento, di arte e verità; un tema, quest'ultimo, già fortemente presente, direi centrale, ne Il sorriso dell'ignoto marinaio.

Ma la scrittura per Vincenzo Consolo non è mai rotonda frontalità espressiva, levigato specchio, ma frantumazione caleidoscopica, allusivo aggiramento, inesauribile nominazione; aldilà della parola, resta, indicibile, il vivido pulsare della vita:

Oh mia Medusa, mia Sfinge, mia Europa, mio sogno e mio pensiero, cos’è mai questa terribile, meravigliosa e oscura vita, questo duro enigma che l'uomo ha sempre declinato in mito, in racconto favoloso e leggendario, per cercar di rispecchiarla, di decifrarla per allusione, per metafora? ${ }^{10}$

Per rappresentare il delirio amoroso di Isidoro, in Retablo, lo scrittore fa perciò interferire iperletterarietà e leggerezza, ricerca espressiva e liricità; scava infatti nelle sonorità del nome Rosalia, trovando in esso occultati tutti i sensi e i segni della passione. Ognuna delle due parti del nome genera infatti una appassionata proliferazione di figure d'amore; se "Rosa» è l'immaginifica sorgente di tutti fiori, i colori, gli aromi, di tutte le sfumature di bellezza dell'amata:

Rosa che ha inebriato, rosa che ha confuso, rosa che ha sventato, rosa che ha ròso, il mio cervello s'è mangiato. Rosa che non è rosa, rosa che è datura, gelsomino, bàlico e viòla; rosa che è pomelia, magnolia, zàgara e cardenia,... Rosa che punto m'ha, ahi!, con la sua spina velenosa in su nel core. ${ }^{11}$

il «li» di «Lia» invece si moltiplica in una spirale di indicibili tormenti amorosi:

Lia che m'ha liato la vita come il cedro o la lumia il dente, liana di tormento, catena di bagno sempiterno, libame oppioso, licore affatturato, letale pozione, lilio dell'inferno che credei divino, lima che sordamente mi corrose l'ossa, limaccia che m'invischiò nelle sue spire, lingua che m'attassò come sangue che guizza dal pietrame, lioparda imperiosa, lippo dell'alma mia, liquame nero, pece dov'affogai, ahi, per mia dannazione. ${ }^{12}$

Il testo non è fine a se stesso, ma fa da attacco al romanzo, immettendo subito il lettore nel cuore tematico del racconto — la vita, l'amore, l'arte - ; presentando i protagonisti — Isidoro, Rosalia, Fabrizio Clerici — ; e profilando l'antefatto e i fatti.

Già nel libro d'esordio - La ferita dell'aprile — lo scrittore apre la narrazione con una una bellissima prosa, scandita dal ritmo dell'endecasillabo: il ricordo del protagonista che rivede se stesso adolescente, in viaggio verso la vita e la consapevolezza storica: 
Dei primi due anni che passai a viaggiare mi rimane la strada arrotolata come un nastro, che posso svolgere: rivedere i tornanti, i fossi, i tumuli di pietrisco incatramato, la croce di ferro passionista, sentire ancora il sole sulla coscia, l'odore di beccume, la ruota che s'affloscia, la naftalina che svapora dai vestiti. ${ }^{13}$

Una lirica dilatazione memoriale, che, con un procedimento tipico della poesia contemporanea, a volte si traduce in contratta densità metaforica, tesa al coinvolgimento emozionale e interpretativo del lettore: esemplare è nello stesso libro la descrizione del panico dei viventi, e della natura tutta, di fronte all'eruzione:

La strada è occhi grandi, dilatati, fronti pesanti sull'arco delle sopracciglia, gambe invischiate lente a trascinarsi, schiene ricurve sotto il cielo basso, la mano gonfia con le dita aperte; il gallo sul pollaio che grida per il nibbio, e il cane che risponde petulante. Il cane e un altro cane e tutti i cani: sì, si spaccò l'Etna. ${ }^{14}$

La presenza di incipit di poesia - in funzione lirica o tragica - aumenta sempre più, libro dopo libro, in funzione di anticipazione metaforica dello sviluppo narrativo, come il testo, tutto assonanze e rime, che da' inizio al capitolo quarto di Nottetempo casa per casa:

Nella vaghezza sua, nell'astrattezza, nella sublime assenza, nella carenza di ragione, di volere, nell'assoluta indifferenza, nel replicare cieco, nella demen$\mathrm{za}$, rivolge a un luogo solo la dura offesa, strema la tenerezza, frange il punto debole, annienta. ${ }^{15}$

Nella risuonante atmosfera che la rima in «enza», scandendo la prosa, genera, s'imprime tragico e dissonante il conclusivo, "Crudo o Vile o Nulla, vuoto vorticoso che calamita, divora, riduce a sua immagine, misura».

Nella produzione consoliana degli anni novanta, una visione sempre più pessimistica della storia tende a instaurare un rapporto inversamente proporzionale tra la rappresentazione della fine di un mondo a carattere antropocentrico e il vitalismo del linguaggio, in funzione di una strutturazione poematica del romanzo, che procede per accumuli lessicali, per addensamenti sonori di consonanze, rime, per grovigli metrici, settenari, novenari, decasillabi, endecasillabi soprattutto.

Lo spostamento in senso poematico della sua narratività è l'esito ultimo di sua riflessione critica sul rapporto scrittura-realtà, che avviene nella seconda metà degli anni ottanta: da un lato - scrive in Fuga dall'Etna — in concomitanza con il «clima spensierato, cinico, di ottuso scialo» della situazione politica italiana di quegli anni — «un carnevale o un paese della cuccagna alla

13. Vincenzo Consolo, La ferita dell'aprile, Torino: Einaudi, 1977, p. 3.

14. Ibid., p. 69.

15. Vincenzo Consolo, Nottetempo casa per casa, Milano: Mondadori, 1992, p. 41. 
Brughel», che lascerà, però, "un campo di macerie»; ${ }^{16}$ dall'altro con la scrittura teatrale di Lunaria e di Catarsi.

In entrambe le opere i versi si alternano al dialogo: una distinzione puramente formale, anzi grafica, perché nella loro testualità non c'è alcuno scarto tra versi e prosa; esemplare è l'attacco in prosa - in realtà in endecasillabi del primo scenario di Lunaria: «E' vasto il vasto regno della Spagna vasto come i castelli di Castiglia, va oltre il mare, s'espande miglia e miglia...». ${ }^{17}$

In Lunaria - una favola teatrale ambientata in una Palermo settecentesca - lo scrittore procede in un'ardita sperimentazione linguistica, portando, soprattutto nelle parti in versi affidate al coro, fino al non sense il gioco linguistico: attraverso ripetizioni consonantiche o sillabiche (ad esempio la funzione della consonante «n», e della vocale «o», in questi versi: «Nutta, nuce, melanì/ voto, ovo sospeso,/ immoto»); ${ }^{18}$ attraverso l'uso della rima, che spesso si ripete — la stessa — verso dopo verso, facendo assumere alle immagini quasi la ritmicità di una giocosa litania, «minna d'innocenti/ melassa di potenti/ tana di briganti, tregua di furfanti»; ${ }^{19}$ e spesso anche attraverso un uso puramente sonoro del significante, che azzera il significato; gioco, ironia, divertissement, che l'uso di un lessico alto, ricercatissimo, commisto di aulicità, dialetto, di termini derivati da tutti i saperi e da tutte le lingue, morte e vive, accentua, «luna, lucore,/ allume lucescente/ levia particula/ fiore albicolante»). ${ }^{20} \mathrm{Ma}$ il risuonante non sense delle cantilene di Lunaria, nulla ha a che fare con il linguaggio afasico della neoavanguardia, esattamente speculare all'afasia del potere per Vincenzo Consolo; nella sua poesia c'è sempre una parola, un verso, una soluzione espressiva che effrange il seduttivo canto di sirena del puro significante. La metamorfosi del protagonista in luna è, ad esempio, una metamorfosi anche linguistica: il vicerè viene come avvolto da un velame di «l»e di «u» di luna, "Lena lennicula,/lemma lavicula,/ làmula/ lémura, mamula./ Létula/ màlia,/ Mah.», ${ }^{21}$ un "mah» finale di una trasgressiva sonorità rispetto ai versi precedenti; quel suono però in persiano significa luna, in arabo vuol dire acqua, mentre in italiano rimanda al dubbio, alla perplessità, e anche alla forma tronca della parola madre. Luna, acqua, dubbio, madre: occultati e oscuramente vibranti nel suono di quel monosillabo.

In Catarsi, attraverso il dramma di un Empedocle contemporaneo - il direttore di un centro di ricerca, che, coinvolto in uno scandalo, è in procinto di buttarsi nell'Etna - lo scrittore invece indaga il rapporto tra parola e realtà nella società contemporanea.

Sul tema della comunicazione infatti drammaticamente si confrontano il protagonista, Empedocle, e l'antagonista, Pausania, che, con la forza persuasiva della parola vorrebbe farlo desistere, rivendicando il suo ruolo di anghelos, di

16. Op. cit., p. 61.

17. Vincenzo Consolo, Lunaria, Torino: Einaudi, 1985, p. 7.

18. Ibid., p. 5.

19. Ibid., p. 6.

20. Ibid., p. 49.

21. Op. cit., p. 21. 
"colui che conosce i nessi, la sintassi, le ambiguità, le astuzie della prosa, del linguaggio»; ;2 ma nella situazione estrema in cui Empedocle si trova nessuna parola può raggiungerlo, "Se le parole si fanno prive di verità, di dignità, di storia, prive di fuoco e suono, se ci manca il conforto loro, non c'è che l'afasia. Non c'è che il buio della mente, la notte della vita." ${ }^{23}$ dice infatti a conclusione della scena terza.

Nell'introduzione a Oratorio (Manni, 2002) — che oltre alla ripubblicazione di Catarsi, comprende anche L'ape iblea (elegia per Noto) del 1998 - in riferimento allo scritto teatrale del 1989, Vincenzo Consolo ribadisce e approfondisce la sua riflessione teorica. Verificando nella contemporaneità gli elementi costitutivi della tragedia greca, afferma che, oggi, non c'è legittimità d'esistenza per l'Anghelos, - il comunicatore che, narrando l'antefatto agli spettatori seduti nella cavea, dava inizio alla messinscena tragica — perché

ormai la cavea è vuota, deserta. Sulla scena è rimasto solo il coro, il poeta che in tono alto, lirico, in una lingua non più comunicabile commenta e lamenta la tragedia senza soluzione, la colpa, il dolore senza catarsi. ${ }^{24}$

Nell'assenza di ascolto, di referente, nel tempo «dell'assoluta insonorità di un contesto istituzionale», s'interrompe ogni rapporto di transitività tra realtà e scrittura: orfana, senza oggetto, davanti alla violenza senza riscatto della storia, al sonno senza risveglio della ragione, la parola non può più disporsi in racconto, ma con una lingua "estrema e dissonante» squarciare la testualità narrativa, destrutturarla, spostando il romanzo «sempre più verso la parte espressiva, la parte poetica».

In realtà non si tratta di una radicale innovazione, piuttosto dello sviluppo di elementi espressivi di poesia — il lamento e l'invettiva — già presenti nei suoi romanzi, benchè con un segno ideologico diverso. A conclusione storica de La ferita dell'aprile, si leva una voce dal fondo della narrazione, che da una più alta prospettiva di giustizia e di pietà, rappresenta con grande forza poetica la strage di Portella della Ginestra del 1 maggio del 1947:

N'ammazzarono tanti in uno spiazzo (c'erano madri e c'erano bambini), come pecore chiuse nel recinto, sprangata la portella. Girarono come pazzi in cerca di riparo ma li buttò buttò buttò riversi sulle pietre una rosa maligna nel petto e nella tempia: negli occhi un sole giallo di ginestra, un sole verde, un sole nero di polvere di lava, di deserto. Disse una vecchia, ferma, i piedi larghi piantati sul terreno: - Femmine, che sono sti lamenti e queste grida con la schiuma in bocca?. Non è la fine: sparagnate il fiato e la vestina per quella manica di morti che verranno appresso. ${ }^{25}$

22. Vincenzo Consolo, Catarsi in Trittico (Bufalino, Consolo, Sciascia), Catania: Sanfilippo, 1989 , p. 21.

23. Ibid., p. 25.

24. Vincenzo Consolo, Oratorio, Lecce: Manni, 2002, p. 6.

25. Op. cit., p. 122-123. 
un requiem per i morti di Portella, in cui però alla rappresentazione della violenza della storia si contrappone quella di una ostinata resistenza ad essa, attraverso la plastica immagine della vecchia con le gambe ben piantate nel terreno.

Snodo poetico e ideologico del libro, ne Il sorriso dell'ignoto marinaio, «il canto lamentoso, il pianto rotto, il cordoglio», riguardano la rivolta e la strage dei braccianti ad Alcara Li Fusi, e la fine della speranza di giustizia sociale che in Sicilia motivò l'adesione popolare al processo unitario ( Sì, bisogna scappare, nascondersi. Bisogna attendere, attendere fermi, immobili, pietrificati»); ${ }^{26}$ ma anche, a mio parere, nascono dalla necessità dello scrittore di dare espressione allo spessore di sofferenza delle tante jaqueries, anonime e inespresse nella grande storia.

Il brano oltrepassa la connotazione linguistica ottocentesca del personaggio del Mandralisca: è la voce dell'autore che, con un ritmo metaforico accelerato, entra in scena a indicare la cieca notte della storia "all'estremo della notte già le orde picchiano alle porte, sgangherano e scardinano con calci chiodati, lasciano croci di gesso su bussole e portelli»): ${ }^{27}$ notte di morti, spari, inseguimenti, ma anche di una possibile sortita verso la luce,

Muovi il tuo piede qui, su questa terra, entra, fissa la scena; in questo spazio invaso dalla notte troverai i passaggi, le fughe, esci se puoi dalla maledizione della colpa, senti: il rantolo tremendo si snoda in prospettiva, mantegnesco. ${ }^{28}$

In Retablo, invece, il lamento diventa invettiva contro «il secol nostro superbo di conquiste e di scienza, secolo illuso, sciocco e involuto.», e contro la città che nel Settecento meglio lo rappresentava - e ancora oggi meglio rappresenta il secolo — l'«attiva, mercatora», Milano,

stupida e volgare mia città che ha fede solamente nel danee, ove impera e trionfa l'impostore, il bauscia, il ciarlatan, il falso artista, el teatrant vacabìnt e pien de vanitaa, il governatore ladro, il prete trafficone, il gazzettier potente, il fanatico credente e il poeta della putrida grascia brianzola; ${ }^{29}$

città che ritorna, oggetto di un'invettiva ancora più amara e disperata, ne Lo Spasimo di Palermo: simbolo dell' «illusione infranta», del disastro storico e morale dell'Occidente,

città perduta, città irreale, d'ombre senz'ombra che vanno e vanno sopra ponti, banchine della darsena, mattatoi e scali, sesto e cinisello disertate, "tennologico» ingranaggio, dallas dello svuotamento e del metallo. Addio. ${ }^{30}$ 
Dopo Nottetempo casa per casa l'invettiva e il lamento si amplificano, acquistando una totale autonomia rispetto alla narrazione: diffusa voce extranarrante, inclusiva di vicende e destini, che, rispondendo a un consapevole disegno poematico del romanzo, blocca gli eventi, immobilizza il racconto senza possibilità di dialettico sviluppo e di autonoma parola nell'afasia della società di oggi.

Ne L'olivo e l'olivastro cade infatti ogni steccato di genere tra poesia e prosa, tra poema e romanzo; il rimando da capitolo a capitolo - le tappe di una contemporanea odissea - non risponde né alla sequenzialità narrativa, né tantomeno a un'organizzazione saggistica. Un'angolazione di poesia fa visionariamente implodere la simultaneità dei tempi della lingua nella sequenzialità di quello narrativo, connotando espressivamente eventi e personaggi. I luoghi si verticalizzano, materializzando - in un continuum di passato e presente, di bellezza e desolazione - la densità di storia e di vissuto che vibra oscurata dietro ogni paesaggio, ogni muro, ogni piazza, ogni città; il viaggio di Odisseo-Consolo verso l'Itaca-Sicilia non è infatti un viaggio «in una dimensione orizzontale. Ma, una volta immerso nella vastità del mare, è come fosse il suo un viaggio in verticale, una discesa negli abissi, nelle ignote dimore, dove a grado a grado, tutto diventa orrifico, subdolo, distruttivo", ${ }^{31}$ dimensione di struggente lirismo si alterna a una poesia di dolente espressività: alla restituzione lirica di poche oasi di civiltà e di memoria - Siracusa, Cefalù, Caltagirone - si contrappone quella, indignata e drammatica, di una degradata contemporaneità, che trova tremenda esemplificazione in Gela, città «della perdita d'ogni memoria e senso, del gelo della mente e dell' afasia, del linguaggio turpe della siringa e del coltello, della marmitta fragorosa e del tritolo», ${ }^{32}$ simbolo dell'irreversibile fine di un'Atene civile, «che - scrive Vincenzo Consolo nel lamento-invettiva di grande forza tragica, a conclusione morale del libro — nessuno può liberare dall'oltraggio». Lo scarto tra parola e realtà, tra racconto e afasia, si acutizza ne Lo spasimo di Palermo, in cui una lingua vertiginosamente espressionista disarticola l'apparenza di romanzo in gorghi di immagini, assonanze, rime, enjambement. Il libro si apre infatti con una sorta di proemio in cui lo scrittore addensa il senso e la connotazione metaforica della sua scrittura («Avanzi per corridoi d'ombre, ti giri e scorgi le tue orme. Una polvere cadde sopra gli occhi, un sonno nell'assenza. Il fumo dello zolfo serva alla tua coscienza. Ora la calma t'aiuti a ritrovare il nome tuo d'un tempo, il punto di partenza»): ${ }^{33}$ un criptico viatico di poesia per il lettore, che insieme al protagonista - trasparente doppio dell'autore - sta intraprendendo un viaggio nell'immobilità della storia e nella fascinazione maliosa ma non consolatoria della parola.

Quasi tutti gli undici capitoli in cui è diviso il romanzo sono introdotti da brevi preludi di un'immaginifica ed enigmatica densità, del tutto irrelati rispetto alla narrazione; un solo esempio: il preludio del IV capitolo, 
Muro che crolla, interno che si mostra, fuga affannosa, segugio che non molla, antro fra ruderi sferzato dalla pioggia, ironiche statue in prospettiva, teschi sui capitelli, maschere sui bordi delle fosse, botteghe incenerite, volumi che in mano si dissolvono, lei al centro d'un quadrivio accovacciata, lei distesa nella stanza che urla e che singhiozza, ritorna dall' estrema soglia, dall'insulinico terrore, entra ed esce per la porta sull'abisso, il tempo è fisso nel continuo passaggio, nell'assenza, nel fondo sono le sequenze, i nessi saldi e veri. ${ }^{34}$

Sono i misteri dolorosi di una via crucis, che, sviluppandosi capitolo dopo capitolo, tocca tutte le stazioni della contemporaneità - dal terrorismo alla speculazione edilizia, alla mafia, alla guerra, alla tenebra della follia, alla condizione di emarginazione urbana «degli stanziali dei margini» — i poveri, i disperati, i migranti — che vivono, oscuri e oscurati, negli squallidi anfratti delle città contemporanee,

Stanno nel tempo loro, nell'immota notte, chiusi nel sudario bruno, ermetici e remoti, stanno come vessilli gravi sui confini, nel passo breve tra il moto e la paralisi. Proni, supini, acchiocciolati contro balaustre, muri, statue in volute di drappi, spiegamento d'ali, slanci fingono l'estro, sono la massa ironica contro illusioni, inganni, monito dell'esito, del lento sfaldamento. ${ }^{35}$

L'intervento poetico e tragico dello scrittore spesso taglia verticalmente la narrazione, assorbendo il narrato nella restituzione metaforica di una condizione storica dove ogni giustizia è morta, ogni pietà s'è spenta.

Sulla stasi e il silenzio della storia («Solca la nave la distesa piana, la corrente scialba, tarda veleggia verso il porto fermo, le fantasime del tempo. La storia è sempre uguale»), ${ }^{36}$ si stende il requiem della poesia: rito di morte e, insieme, esorcismo contro la morte, di una scrittura che sul ciglio degli abissi «si raggela, si fa suono fermo, forma compatta, simbolo sfuggente»; 37 barocca fascinazione tonale di un linguaggio risuonante di rime, nominazioni, fastose metafore, che, simultaneamente, si pone come emergenza espressiva, ed estremo gesto di libertà ideologica, in una condizione umana coatta dalle istituzioni di potere e dall'assertorietà definitoria, ma anch'essa ideologica, del linguaggio, "La lingua - dice Vincenzo Consolo citando in un articolo della rivista Autodafe Roland Barthes — non è né reazionaria, né progressista: è semplicemente fascista, il fascismo non è impedire di dire, ma obbligare a dire...».

Non resta allora che l'afasia o la poesia. E Vincenzo Consolo sceglie la poesia.

34. Ibid., p. 45.

35. Ibid., p. 70-71.

36. Ibid., p. 9.

37. Ibid., p. 12. 